\title{
Mathematical model of non-stationary temperature distribution in the metal body produced by induction heating process
}

\author{
Josef $\operatorname{Rak}^{1,2, a}$
}

1 University of Pardubice, Faculty of Electrical Engineering and Informatics, Studentska 9553210 Pardubice, Czech Republic

2 Some results were obtained by author's doctoral study in Charles University in Prague, Faculty of Mathematics and Physics, Department of Numerical Mathematics

\begin{abstract}
An induction heating problem can be described by a parabolic differential equation. For this equation, specific Joule looses must be computed. It can be done by solving the Fredholm Integral Equation of the second kind for the eddy current of density. When we use the Nyström method with the singularity subtraction, the computation time is rapidly reduced. This paper shows the method for finding non-stationary temperature distribution in the metal body with illustrative examples.
\end{abstract}

Key words. induction heating, integral equation of the second kind, non-stationary temperature distribution, Nyström method

\section{Introduction}

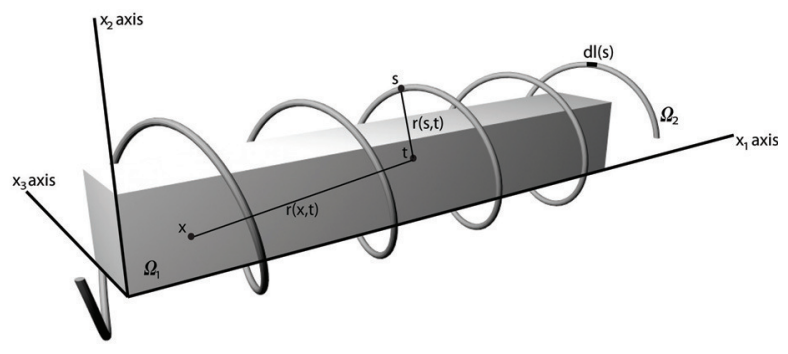

Figure 1. Heated body and coil.

A bounded metal cuboid body $\Omega_{1}$ of sizes $l_{1} \times l_{2} \times$ $l_{3}$ is heated by an external electromagnetic field produced by inductor $\Omega_{2}$ (see figure 1). The inductor is formed by a conductor of general shape and position that carries the harmonic current $I_{\text {ext }}$.

\section{Eddy current of density}

Computation of the temperature distribution depends on the eddy current of density. It is a phasor

$$
J_{e d d y}=\left(J_{e d d y, x_{1}}, J_{e d d y, x_{2}}, J_{e d d y, x_{3}}\right) .
$$

The $x_{1}$ component of $J_{e d d y, x_{1}}(\boldsymbol{x})$ can be computed (see [4] and [5]) by Fredholm integral equation of the second kind

$$
\iota J_{e d d y, x_{1}}(\boldsymbol{x})-\kappa(\boldsymbol{x}) \int_{\Omega_{1}} \frac{J_{e d d y, x_{1}}(\boldsymbol{t})}{r(\boldsymbol{x}, \boldsymbol{t})} d t_{1} d t_{2} d t_{3}=I_{e x t} F(\boldsymbol{x}),
$$

\footnotetext{
${ }^{\text {a }}$ Corresponding author: josef.rak@upce.cz
}

where

$$
\begin{gathered}
F(\boldsymbol{x})=\kappa(\boldsymbol{x}) \int_{\Omega_{2}} \frac{d l(\boldsymbol{s}) \cdot e_{x_{1}}}{r(\boldsymbol{x}, \boldsymbol{s})}, \\
\kappa(\boldsymbol{x})=\frac{\omega \gamma(T(\boldsymbol{x})) \mu_{0}}{4 \pi},
\end{gathered}
$$

$r(\boldsymbol{x}, \boldsymbol{t})$ is the Euclidean distance, $\boldsymbol{x}=\left(x_{1}, x_{2}, x_{3}\right)$ and $\boldsymbol{t}=$ $\left(t_{1}, t_{2}, t_{3}\right)$ are points in the metal body, $\boldsymbol{s}=\left(s_{1}, s_{2}, s_{3}\right)$ is a point at the inductor, $I_{\text {ext }}$ is harmonic current carried by the inductor, $\omega$ is angular frequency, $\gamma(T(\boldsymbol{x}))$ is electrical conductivity, $\mu_{0}$ is permeability of vacuum, $e_{x_{1}}$ is unite vector $e_{x_{1}}=(1,0,0)$ and $\iota$ is the complex unit.

For each bounded and continuous temperature distribution $T(\boldsymbol{x}), \kappa(\boldsymbol{x})$ is a real, positive, bounded and continuous function.

Formulas for the remaining components $J_{e d d y, x_{2}}$ and $J_{e d d y, x_{3}}$ can be obtained by mere interchanging of indices.

With the notation

$$
\begin{aligned}
J_{R}(\boldsymbol{x}) & =\operatorname{Re} J_{e d d y, x_{1}}(\boldsymbol{x}) \\
J_{I}(\boldsymbol{x}) & =\operatorname{Im} J_{e d d y, x_{1}}(\boldsymbol{x}) \\
I_{R} & =\operatorname{Re}\left(I_{\text {ext }}\right) \\
I_{I} & =\operatorname{Im}\left(I_{\text {ext }}\right)
\end{aligned}
$$

we have system of integral equations

$$
\begin{aligned}
J_{R}(\boldsymbol{x})-\kappa(\boldsymbol{x}) \int_{\Omega_{1}} \frac{J_{I}(\boldsymbol{t})}{r(\boldsymbol{x}, \boldsymbol{t})} d t & =I_{I} F(\boldsymbol{x}), \\
-J_{I}(\boldsymbol{x})-\kappa(\boldsymbol{x}) \int_{\Omega_{1}} \frac{J_{R}(\boldsymbol{t})}{r(\boldsymbol{x}, \boldsymbol{t})} d t & =I_{R} F(\boldsymbol{x}) .
\end{aligned}
$$

The specific Joule losses at the point $\boldsymbol{x}$ in the body, which are needed to compute the temperature distribution are given by

$$
\omega(\boldsymbol{x})=\frac{1}{\gamma} J_{e}(\boldsymbol{x}) \overline{J_{e}(\boldsymbol{x})}
$$


where

$$
J_{e}(\boldsymbol{x})=
$$

$$
\begin{gathered}
\sqrt{\left[\operatorname{ReJ}_{e d d y, x_{1}}(\boldsymbol{x})\right]^{2}+\left[\operatorname{ReJ}_{e d d y, x_{2}}(\boldsymbol{x})\right]^{2}+\left[\operatorname{ReJ}_{e d d y, x_{3}}(\boldsymbol{x})\right]^{2}}+ \\
+\iota \sqrt{\left[\operatorname{ImJ}_{e d d y, x_{1}}(\boldsymbol{x})\right]^{2}+\left[\operatorname{ImJ}_{e d d y, x_{2}}(\boldsymbol{x})\right]^{2}+\left[\operatorname{ImJ}_{e d d y, x_{3}}(\boldsymbol{x})\right]^{2}}
\end{gathered}
$$

and $\overline{J_{e}(\boldsymbol{x})}$ is complex conjugate to $J_{e}(\boldsymbol{x})$.

\section{The non-stationary temperature distrubution}

The non-stationary distribution of the temperature $T(x, t)$ at point $\boldsymbol{x}$ and time $t$ in the metal body is described by a partial differential equation of the parabolic type

$$
\begin{gathered}
\operatorname{div}[\lambda(T(\boldsymbol{x}, t)) \operatorname{grad} T(\boldsymbol{x}, t)]= \\
=\rho(T(\boldsymbol{x}, t)) c(T(\boldsymbol{x}, t)) \frac{\partial T(\boldsymbol{x}, t)}{\partial t}-\omega(\boldsymbol{x}, t),
\end{gathered}
$$

where $\lambda=\lambda(T(\boldsymbol{x}, t))$ denotes the thermal conductivity, $\rho=$ $\rho(T(\boldsymbol{x}, t))$ the specific mass of the material, $c=c(T(\boldsymbol{x}, t))$ specific heat and $\omega(\boldsymbol{x}, t)$ the specific Joule losses given by (9) at time $t$.

The boundary condition along the whole surface of the body reads

$$
-\lambda(T(\boldsymbol{x}, t)) \frac{\partial T(\boldsymbol{x}, t)}{\partial n}=\alpha\left(T(\boldsymbol{x}, t)-T_{e x t}\right),
$$

where $\alpha$ denotes the coefficient of the convective heat transfer, $T_{\text {ext }}$ the temperature of the surrounding medium and $n$ the direction of the outward normal.

\section{Numerical solution}

The metal body $\Omega_{1}$ was assumed to be cuboid. Let us cover the body by $n_{1}$ subsuboides in the $x_{1}$ direction, $n_{2}$ subsuboides in the $x_{2}$ direction and $n_{3}$ subsuboides in the $x_{3}$ direction. The size of each subcuboid is $h_{1} \times h_{2} \times h_{3}$, where

$$
h_{1}=\frac{l_{1}}{n_{1}}, h_{1}=\frac{l_{2}}{n_{2}}, h_{3}=\frac{l_{3}}{n_{3}} .
$$

We need to solve integral equation (1) to get Joule losses. Then we can put computed Joule losses to partial differential equation (10) to get the temperature distribution. The Joule losses must be recalculated at the time when changed.

For computation of Joule losses we will apply the Nyström method with the compound mid-cuboid rule. For time integration we will use the classical finite difference method for partial differential equations of the parabolic type.

\subsection{Application of Nyström method}

The Nyström method is based on approximation of the integral by the numerial integration rule. We will use the compound min-point rule. Let the node points be $\boldsymbol{x}_{i}$ defined as center of sub-cuboides. For the weight let

$$
w_{j}=w=\frac{\left|\Omega_{1}\right|}{N}, j=1, \ldots, N,
$$

where $N=n_{1} n_{2} n_{3}$.

Here the numerical integration rule cannot be applied directly. The reason is that the function $r(x, t)^{-1}$ is singular at $\boldsymbol{x}=\boldsymbol{t}$. One way to deal with such a problem is singularity subtraction. It was described in [3]. Let $r_{N}(\boldsymbol{x}, \boldsymbol{t})$ be an approximation of $r(\boldsymbol{x}, \boldsymbol{t})$ which coincide outside a certain neighborhood of $\boldsymbol{x}=\boldsymbol{t}$. The integrand in equation (1) is approximated by

$$
\frac{J_{e d d y, x_{1}}(\boldsymbol{t})}{r(\boldsymbol{x}, \boldsymbol{t})} \approx \frac{J_{e d d y, x_{1}}(\boldsymbol{t})-J_{e d d y, x_{1}}(\boldsymbol{x})}{r_{N}(\boldsymbol{x}, \boldsymbol{t})}+\frac{J_{e d d y, x_{1}}(\boldsymbol{x})}{r(\boldsymbol{x}, \boldsymbol{t})} .
$$

Since the first element of the approximation is zero when $\boldsymbol{x}=\boldsymbol{t}$, the exact constructioin of $r_{N}(\boldsymbol{x}, \boldsymbol{t})$ is immaterial. By using the numerical integration rule, approximation (12) and running $\boldsymbol{x}$ throung the node points we get a system of linear equations for numerical approximation of the $x_{1}$ component of the eddy current of density $\widetilde{J}_{N}$

$$
\begin{gathered}
{\left[1-\iota \kappa\left(\boldsymbol{x}_{i}\right) \sum_{\substack{j=1 \\
j \neq i}}^{N} \frac{w}{r_{N}\left(\boldsymbol{x}_{i}, \boldsymbol{x}_{j}\right)}=\iota \kappa\left(\boldsymbol{x}_{i}\right) \int_{\Omega_{1}} \frac{d t_{1} d t_{2} d t_{3}}{r\left(\boldsymbol{x}_{i}, \boldsymbol{t}\right)}\right] \widetilde{J}_{N}\left(\boldsymbol{x}_{i}\right)+} \\
\quad+\iota \kappa\left(\boldsymbol{x}_{i}\right) \sum_{\substack{j=1 \\
j \neq i}}^{N} \frac{w \widetilde{J}_{N}\left(\boldsymbol{x}_{j}\right)}{r_{N}\left(\boldsymbol{x}_{i}, \boldsymbol{x}_{j}\right)}=-\iota I_{e x t} F\left(\boldsymbol{x}_{i}\right), i=1, \ldots, N
\end{gathered}
$$

$\widetilde{J}_{N}\left(\boldsymbol{x}_{i}\right)$ is complex number. Let us define for each $i=1, \ldots, N$

$$
\widetilde{J}_{i}^{(R)}=\operatorname{Re} \widetilde{J}_{N}\left(\boldsymbol{x}_{i}\right) \text { and } \widetilde{J}_{i}^{(I)}=\operatorname{Im} \widetilde{J}_{N}\left(\boldsymbol{x}_{i}\right)
$$

Then (13) is equivalent the real system of linear equations

$$
\begin{gathered}
\widetilde{J}_{i}^{(R)}+\kappa\left(\boldsymbol{x}_{i}\right)\left[\sum_{\substack{j=1 \\
j \neq i}}^{N} \frac{w}{r_{N}\left(\boldsymbol{x}_{i}, \boldsymbol{x}_{j}\right)}-\int_{\Omega_{1}} \frac{d t_{1} d t_{2} d t_{3}}{r\left(\boldsymbol{x}_{i}, \boldsymbol{t}\right)}\right] \widetilde{J}_{i}^{(I)}- \\
-\kappa\left(\boldsymbol{x}_{i}\right) \sum_{\substack{j=1 \\
j \neq i}}^{N} \frac{w \widetilde{J}_{j}^{(I)}}{r_{N}\left(\boldsymbol{x}_{i}, \boldsymbol{x}_{j}\right)}=\operatorname{Im} I_{e x t} F\left(\boldsymbol{x}_{i}\right), i=1, \ldots, N \\
-\widetilde{J}_{i}^{(I)}+\kappa\left(\boldsymbol{x}_{i}\right)\left[\sum_{\substack{j=1 \\
j \neq i}}^{N} \frac{w}{r_{N}\left(\boldsymbol{x}_{i}, \boldsymbol{x}_{j}\right)}-\int_{\Omega_{1}} \frac{d t_{1} d t_{2} d t_{3}}{r\left(\boldsymbol{x}_{i}, \boldsymbol{t}\right)}\right] \widetilde{J}_{i}^{(R)}- \\
-\kappa\left(\boldsymbol{x}_{i}\right) \sum_{\substack{j=1 \\
j \neq i}}^{N} \frac{w \widetilde{J}_{j}^{(R)}}{r_{N}\left(\boldsymbol{x}_{i}, \boldsymbol{x}_{j}\right)}=\operatorname{Re} I_{e x t} F\left(\boldsymbol{x}_{i}\right), i=1, \ldots, N .
\end{gathered}
$$

Following Nyström interpolation formula (for details see for example [1]) we get the numerical solution for eddy currents $\widetilde{J}_{N}$ outside the node points

$$
\widetilde{J}_{N}(\boldsymbol{x})=\frac{-\iota I_{e x t} F(\boldsymbol{x})-\iota \kappa(\boldsymbol{x}) \sum_{j=1}^{N} \frac{w}{r_{N}\left(\boldsymbol{x}, \boldsymbol{x}_{j}\right)} \widetilde{J}_{N}\left(\boldsymbol{x}_{j}\right)}{1-\iota \kappa(\boldsymbol{x}) \sum_{j=1}^{N} \frac{w}{r_{N}\left(\boldsymbol{x}, \boldsymbol{x}_{j}\right)}+\iota \kappa(\boldsymbol{x}) \int_{\Omega_{1}} \frac{1}{r(\boldsymbol{x}, \boldsymbol{t})} d \boldsymbol{t}},
$$


where

$$
\widetilde{J}_{N}\left(\boldsymbol{x}_{i}\right)=\widetilde{J}_{i}^{(R)}+\iota \widetilde{J}_{i}^{(I)} .
$$

Since $\Omega_{1}$ is a cuboid, the integral in (15) and (16) can be computed analytically. The $x_{2}$ and $x_{3}$ components of the eddy current of density can be computed in an analogous way.

\section{Application of the finite difference method}

Let us use a finite difference method for the numerical solution of equation (10). Since thermal conductivity is a constant that depends on the temperature we can rewrite equation (10) into the form

$$
\begin{array}{r}
\frac{\partial T(\boldsymbol{x}, t)}{\partial t}=\frac{1}{\rho(T(\boldsymbol{x}, t)) c(T(\boldsymbol{x}, t))}[\lambda(T(\boldsymbol{x}, t)) . \\
\left.\cdot\left(\frac{\partial^{2} T(\boldsymbol{x}, t)}{\partial x_{1}^{2}}+\frac{\partial^{2} T(\boldsymbol{x}, t)}{\partial x_{2}^{2}}+\frac{\partial^{2} T(\boldsymbol{x}, t)}{\partial x_{3}^{2}}\right)+\omega(\boldsymbol{x}, t)\right] .
\end{array}
$$

Let the coordinates of the node point be

$$
\left(x_{1_{i}}, x_{2_{j}}, x_{3_{k}}\right), i=1, \ldots, n_{1}, j=1, \ldots n_{2}, k=1, \ldots, n_{3} .
$$

With the notation

$$
\begin{aligned}
x_{i, j, k} & =x_{1_{1}}, x_{2_{j}}, x_{3_{k}} \\
T_{i, j, k}(t) & =T\left(x_{i, j, k}, t\right) \\
\omega_{i, j, k}(t) & =\omega\left(x_{i, j, k}, t\right)
\end{aligned}
$$

the $x_{1}, x_{2}, x_{3}$ derivatives are approximated by

$$
\begin{aligned}
& \frac{\partial^{2} T(\boldsymbol{x}, t)}{\partial x_{1}^{2}} \approx \frac{T_{i+1, j, k}(t)-2 T_{i j k}(t)+T_{i-1, j, k}(t)}{h_{1}^{2}} \\
& \frac{\partial^{2} T(\boldsymbol{x}, t)}{\partial x_{2}^{2}} \approx \frac{T_{i, j+1, k}(t)-2 T_{i j k}(t)+T_{i, j-1, k}(t)}{h_{2}^{2}} \\
& \frac{\partial^{2} T(\boldsymbol{x}, t)}{\partial x_{3}^{2}} \approx \frac{T_{i, j, k+1}(t)-2 T_{i j k}(t)+T_{i, j, k-1}(t)}{h_{3}^{2}} .
\end{aligned}
$$

Now let us approximate of the derivation by difference in the boudary condition (11) to define $T_{i j k}$ for $i=0, i=$ $n_{1}+1, j=0, j=n_{2}+1, k=0$ and $k=n_{3}+1$ as

$$
\begin{aligned}
T_{0, j, k}(t) & =T_{1, j, k}-\frac{h_{1} \alpha}{\lambda\left(T_{1, j, k}(t)\right)}\left(T_{1, j, k}-T_{e x t}\right) \\
T_{n_{1}+1, j, k}(t) & =T_{n_{1}, j, k}-\frac{h_{1} \alpha}{\lambda\left(T_{n_{1}, j, k}(t)\right)}\left(T_{n_{1}, j, k}-T_{e x t}\right) \\
T_{i, 0, k}(t) & =T_{i, 1, k}-\frac{h_{2} \alpha}{\lambda\left(T_{i, 1, k}(t)\right)}\left(T_{i, 1, k}-T_{e x t}\right) \\
T_{i, n_{2}+1, k}(t) & =T_{i, n_{2}, k}-\frac{h_{2} \alpha}{\lambda\left(T_{i, n_{2}, k}(t)\right)}\left(T_{i, n_{2}, k}-T_{e x t}\right) \\
T_{i, j, 0}(t) & =T_{i, j, 1}-\frac{h_{3} \alpha}{\lambda\left(T_{i, j, 1}(t)\right)}\left(T_{i, j, 1}-T_{e x t}\right) \\
T_{i, j, n_{3}+1}(t) & =T_{i, j, n_{3}}-\frac{h_{3} \alpha}{\lambda\left(T_{i, j, n_{3}}(t)\right)}\left(T_{i, j, 1 n_{3}}-T_{e x t}\right) .
\end{aligned}
$$

Let us define time step $\Delta t$ and approximate time derivation by

$$
\frac{\partial T(\boldsymbol{x}, t)}{\partial t} \approx \frac{T(\boldsymbol{x}, t+\Delta t)-T(\boldsymbol{x}, t)}{\Delta t} .
$$

From (17), (18), (19) and (20) we have numerical formula for temperature evolution

$$
\begin{gathered}
T_{i, j, k}(t+\Delta t)=T_{i, j, k}(t)+\frac{\Delta t}{\left.\rho\left(T_{i, j, k}(t)\right) c\left(T_{i, j, k}(t)\right)\right)} . \\
\cdot\left[\lambda ( T _ { i j k } ( t ) ) \left(\frac{T_{i+1, j, k}(t)-2 T_{i, j, k}(t)+T_{i-1, j, k}(t)}{h_{1}^{2}}+\right.\right. \\
+\frac{T_{i, j+1, k}(t)-2 T_{i, j, k}(t)+T_{i, j-1, k}(t)}{h_{2}^{2}}+ \\
\left.\left.+\frac{T_{i, j, k+1}(t)-2 T_{i, j, k}(t)+T_{i, j, k-1}(t)}{h_{3}^{2}}\right)+\omega_{i, j, k}(t)\right] .
\end{gathered}
$$

Joule losses need to be updated when time changes. The whole calculation is started with an initial condition. It is the starting temperature of the body and is equal to temperature of the air of the surroundig medium.

\section{Example 1}

A brass cuboid body with the size $0.15 \times 0.01 \times 0.01 \mathrm{~m}$ is heated with a stationary inductor starting at room temperature $20^{\circ} \mathrm{C}$. The inductor has the form of a coil which turns around the heated body in the $x_{1}$-direction in 6 loops. The radius of the coil is $0.015 \mathrm{~m}$, exciting current $500 \mathrm{~A}$ and frequency $150 \mathrm{kHz}$. The length of the coil is $0.15 \mathrm{~m}$. The cuboid is partitioned by 75 elements in $x_{1}$ direction, 10 elements in $x_{2}$ and $x_{3}$ directions. Figures 2 - 4 show temperature distribution during time evolution $(1,10$ and $60 \mathrm{~s})$ at the surface of the heating body. Figures $5-7$ show temperature distribution during time evolution $(1,10$ and $60 \mathrm{~s})$ at cuts of the body when $x_{2}=0$ and $x_{3}=0$.

Computation was made by Matlab. The integration for computing $F\left(x_{i}\right)$ is done by the Matlab's method quad. It uses an aplication of the adaptive Simpson quadrature. Improper integral is computed analytically.

\section{Example 2}

To see the dependence of the parameters let us show examples with different parameters. Let the parameters be the same as in example 1. Figure 8 shows temperature distribution at $30 \mathrm{~s}$. Other figures have change in one parameter.

- at figure 9 the coil rotates over the heating body at 3 loops.

- at figure 10 the size of the body is $0.15 \times 0.005 \times 0.005 \mathrm{~m}$.

- at figure 11 the coil is exciting current $1000 \mathrm{~A}$.

- at figure 12 the angular frequency is $300 \mathrm{kHz}$.

\section{Conclusion}

In example 2, the temperature changed as expected. It was shown in [5], that the time to compute the Joule losses is reduced to approximately 10 percent compared to the collocation method. Since the Joule losses need to be recalculated $3-10$ times during time evolation to $60 \mathrm{~s}$ is this method a big improovement. 


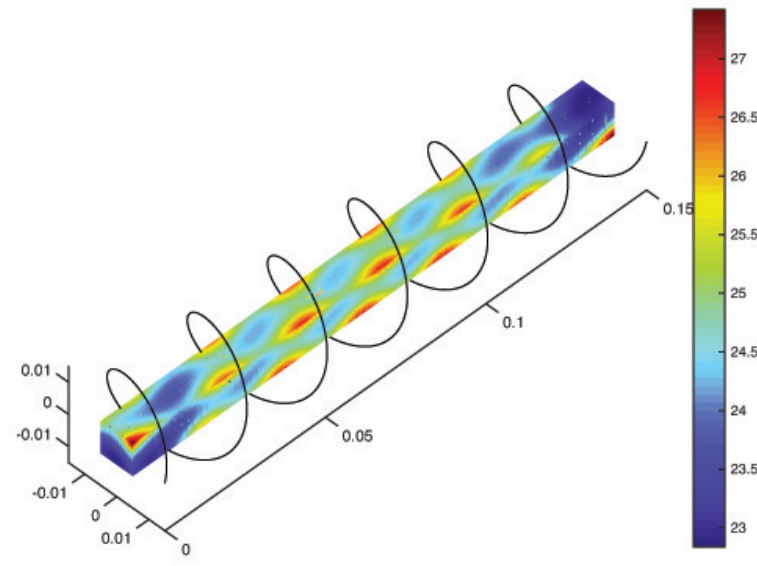

Figure 2. Example 1, $\mathrm{t}=1 \mathrm{~s}$, body

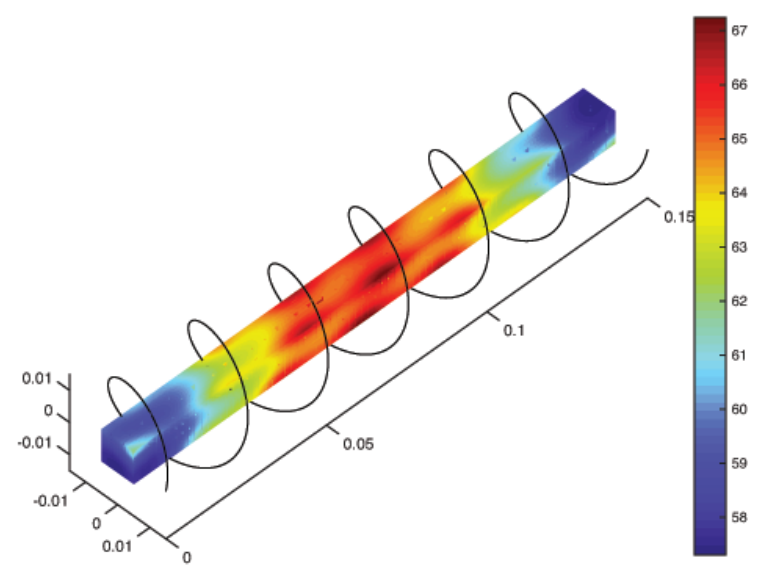

Figure 3. Example 1, $t=10 \mathrm{~s}$, body

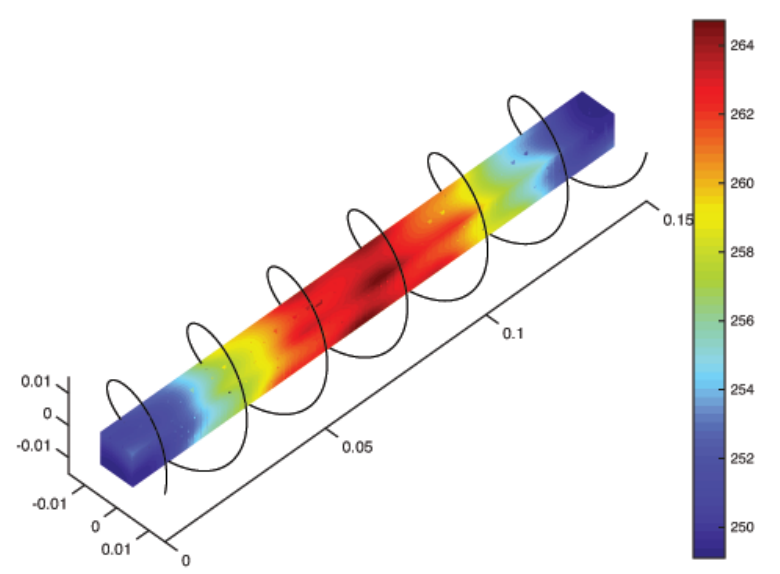

Figure 4. Example 1, $\mathrm{t}=60$ s, body

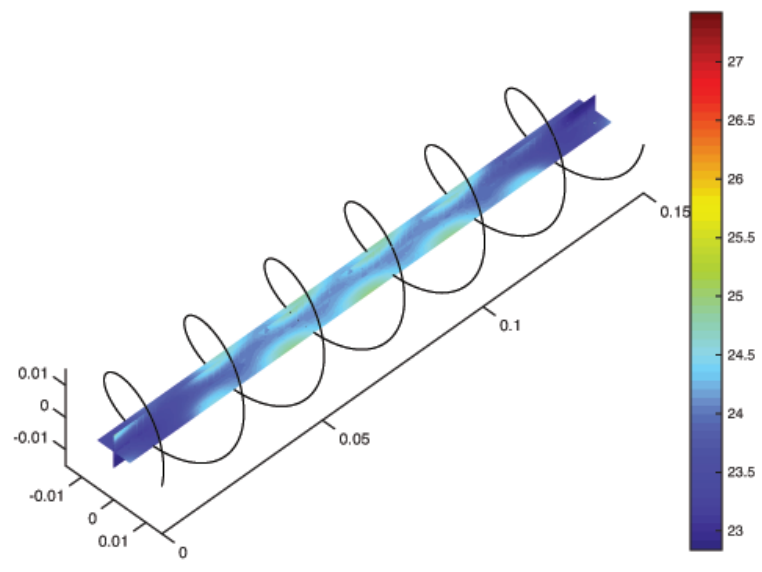

Figure 5. Example 1, $t=1 \mathrm{~s}$, body cut

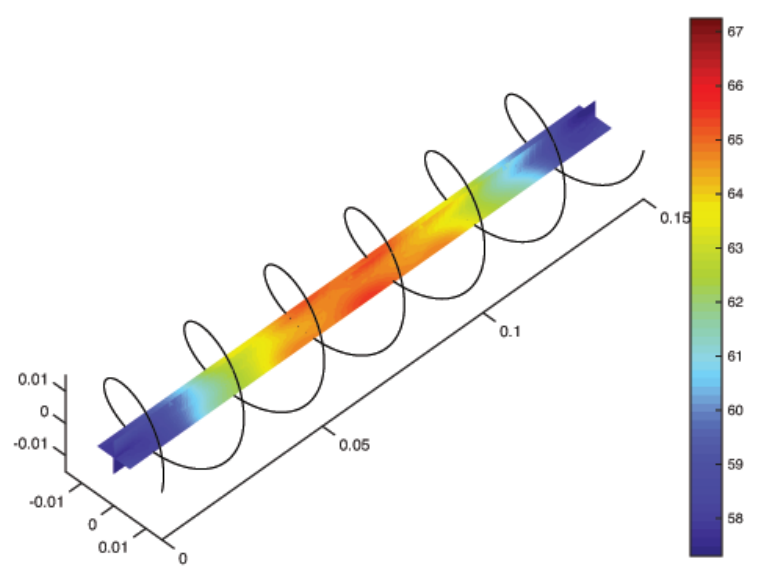

Figure 6. Example 1, $\mathrm{t}=10 \mathrm{~s}$, body cut

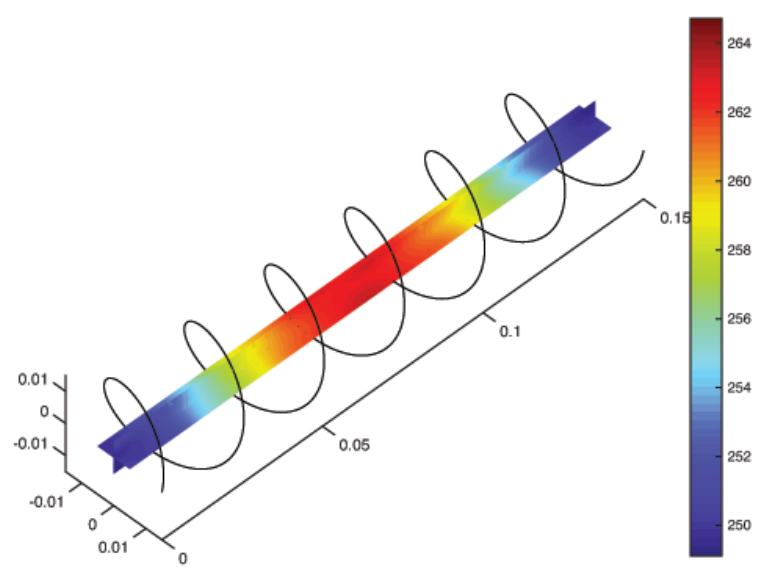

Figure 7. Example 1, $t=60 \mathrm{~s}$, body cut 


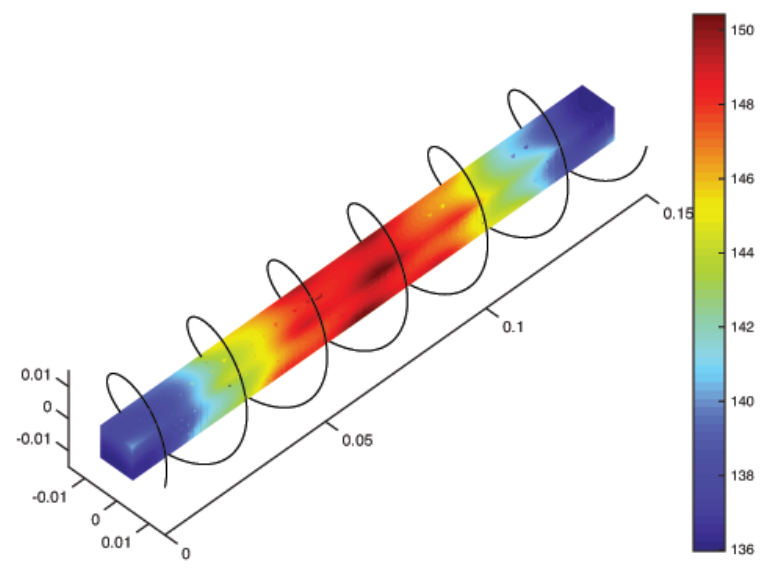

Figure 8. Example 2, $\mathrm{t}=30 \mathrm{~s}$, original parameters

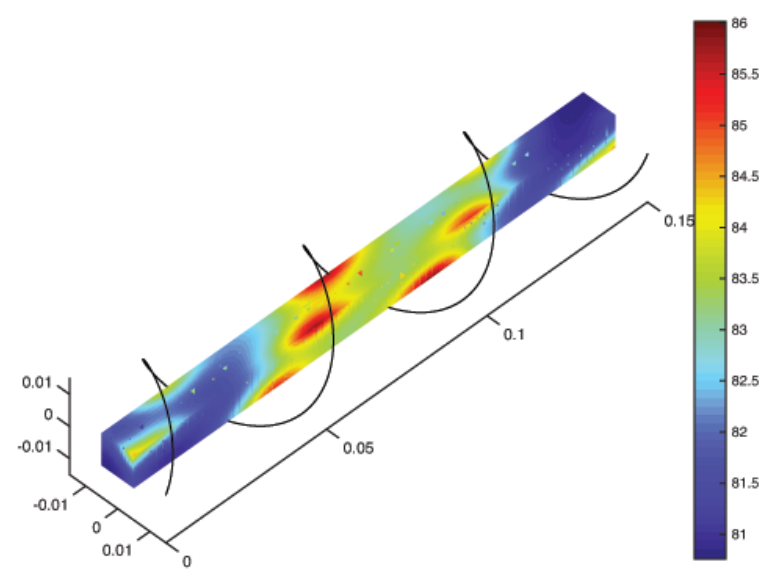

Figure 9. Example 2, $t=30 \mathrm{~s}, 3$ loops of the coil

\section{References}

1. K. E. Atkinson: The Numerical Solution of Integral Equations of the Second Kind (Cambridge University Press, 1997)

2. P. M. Anselone: Collectively compact operator approximation theory and applications to integral equations (Prentice-Hall, Inc., Englewood Cliffs, N.J. 1971)

3. L. V. Kantorovich, V. I. Krylov: Approximate Methods of Higher Analysis, (Interscience, New York, 1958)

4. P. Solin, I. Dolezel, M. Skopek, B. Ulrych, SPETO 2001 International Conference, 143-146 (2001)

5. J. Rak, EPJ web conf. 92, 02075 (2015)

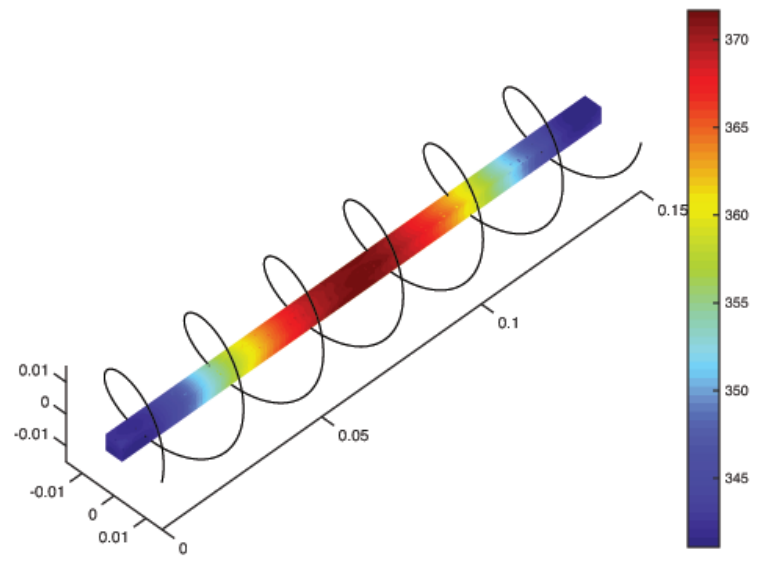

Figure 10. Example 2, $\mathrm{t}=30 \mathrm{~s}$, size $0.15 \times 0.005 \times 0.005 \mathrm{~m}$

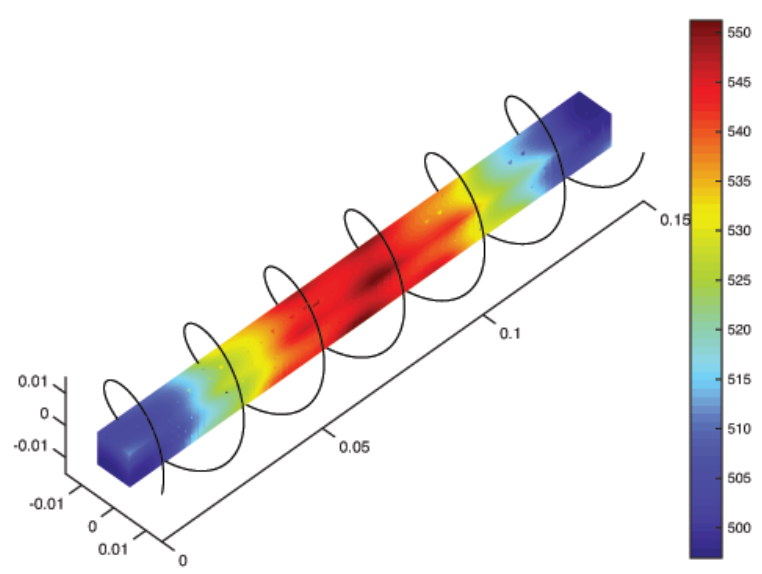

Figure 11. Example 2, $t=30 \mathrm{~s}$, harmonic current $1000 \mathrm{~A}$

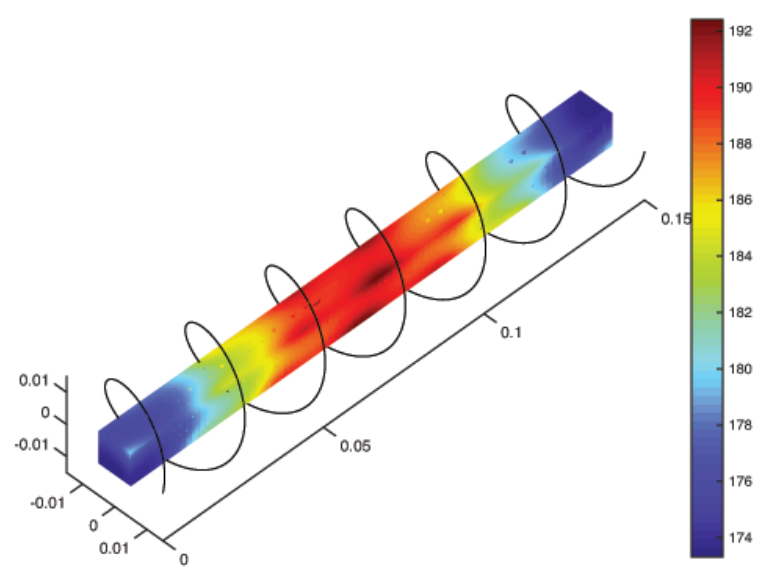

Figure 12. Example 2, $\mathrm{t}=30 \mathrm{~s}$, angular frequency $300 \mathrm{kHz}$ 